Simulation of Explosion Ground Motions Using a Hydrodynamic-to-Elastic Coupling Approach in Three-Dimensions

H. Xu, A. J. Rodgers, I. N. Lomov, N. A. Petersson, B. Sjogreen, O. Y. Vorobiev

May 17, 2012

Bulletin of the Seismological Society of America 
This document was prepared as an account of work sponsored by an agency of the United States government. Neither the United States government nor Lawrence Livermore National Security, LLC, nor any of their employees makes any warranty, expressed or implied, or assumes any legal liability or responsibility for the accuracy, completeness, or usefulness of any information, apparatus, product, or process disclosed, or represents that its use would not infringe privately owned rights. Reference herein to any specific commercial product, process, or service by trade name, trademark, manufacturer, or otherwise does not necessarily constitute or imply its endorsement, recommendation, or favoring by the United States government or Lawrence Livermore National Security, LLC. The views and opinions of authors expressed herein do not necessarily state or reflect those of the United States government or Lawrence Livermore National Security, LLC, and shall not be used for advertising or product endorsement purposes. 


\title{
Simulation of Explosion Ground Motions Using a Hydrodynamic-to-Elastic Coupling Approach in Three-Dimensions
}

\author{
Heming Xu ${ }^{1}$, Arthur J. Rodgers ${ }^{1}$, Ilya N. Lomov ${ }^{1}$, N. Anders Petersson ${ }^{2}$, \\ Bjorn Sjogreen ${ }^{2}$ and Oleg Y. Vorobiev ${ }^{1}$ \\ ${ }^{1}$ Atmospheric, Earth and Energy Division, Physical and Life Sciences Directorate \\ ${ }^{2}$ Center for Advanced Scientific Computing, Computations Directorate \\ Lawrence Livermore National Laboratory, Livermore, CA 94551 USA
}

Manuscript in preparation for the Bulletin of the Seismological Society of America

May 16, 2012

\begin{abstract}
Explosion motions in the solid earth or atmosphere are governed by hydrodynamics and must include non-linear material response effects. However, the numerical calculation of the hydrodynamic response is computationally intensive compared to linear (e.g. elastic and acoustic) wave propagation solvers due to non-linear constitutive behavior especially. In order to propagate explosion generated ground motions from the non-linear near-source region to the far-field we developed a hybrid modeling approach with one-way hydrodynamic-to-elastic coupling in three dimensions. Near source motions are computed with GEODYN, an Eulerian hydrodynamics code with adaptive mesh refinement for high-energy loading of earth materials. Motions on a dense grid of points are saved, resampled and then passed to WPP, an anelastic finite difference code for seismic wave modeling. Our coupling strategy is based on the uniqueness theorem where motions are introduced into WPP as a boundary source and continue
\end{abstract}


to propagate as elastic waves at much lower computational cost than with GEODYN. We have developed and verified the methodology to compute GEODYN hydrodynamic responses in either two- or three-dimensions (2D and 3D) and pass these to WPP as 3D boundary motions on the faces of a cube. For the 2D case we compute the axisymmetric response with GEODYN and transform the elastic motions onto the cube faces (coupling interface) in 3D before introducing motions to WPP. The accuracy of the numerical calculations and the coupling strategy is demonstrated in cases with a purely elastic medium as well as non-linear medium. Importantly we show that GEODYN can accurately model motions for a linear elastic medium including surface waves, which is essential to insure that near-source motions are correct. An application of our hybrid modeling approach is shown for a problem involving scattering by 3D heterogeneity. Our strategy by design is capable of incorporating complex non-linear effects near the source as well as volumetric and topographic material heterogeneity along the propagation path to receiver, making it very powerful for modeling a wide variety of effects and providing new prospects for modeling and understanding explosion generated seismic waveforms.

\section{Introduction}

Improved understanding of explosion generated mechanical motions through numerical modeling helps advance interpretation of seismic data for nuclear explosion monitoring (NEM) and explosion forensics. Currently, there are several significant challenges for the monitoring community involving understanding the effects of emplacement material properties, damage, pre-stress and near-source heterogeneity (volumetric and topographic) on wave motion 
amplitudes and the generation and partitioning of energy into different modes (e.g. compressional and shear waves, body- and surface waves). Understanding these phenomena will improve yield estimation and event identification by accounting for predictable effects on wave motions due to source emplacement, near-source heterogeneity and path-specific propagation effects. Knowledge gained on these effects can then be applied to regions where no empirical data exist, either in regions without historical explosion sources or seismic recordings or for explosions conducted under uncalibrated emplacement conditions (e.g. different media, depth-ofburial or yield).

Numerical simulations provide a versatile tool to gain insight into the generation and propagation of wave motions including both non-linear and linear effects. Explosions are well known to involve the near instantaneous release of high temperature and pressure gas in a small volume of space. These high energy densities cause irreversible non-linear behavior in the surrounding material (rock) due to the generation and propagation of the outgoing hydrodynamic shock wave. It has been long appreciated that non-linear response effects (e.g. material strength, damage) at the explosion emplacement have a strong impact on the observed far-field seismic motions (e.g. Werth and Herbst, 1963; Perret and Bass, 1975; Rodean, 1981; Murphy, 1981; Denny and Johnson, 1991). However, a full understanding of these effects has been hampered by limitations in the knowledge of and computational strategies to represent all relevant non-linear material response effects and to propagate waves from the source region to receivers. Advances in numerical methods and more powerful computational resources now make it possible to routinely compute the hydrodynamic response of earth materials to buried explosions with ever improving fidelity (e.g. Rodgers et al., 2008, 2009ab). Rougier et al. (2011) used hydrodynamic 
modeling to constrain the trade-off between yield and depth-of-burial for the May 25, 2009 North Korean nuclear explosion. Recently, Xu et al. (2011) showed that hydrodynamic modeling of buried explosions in a well-calibrated granite material model reproduces the elastic source spectra predicted by the widely accepted empirical source model of Mueller and Murphy (1971) and Stevens and Day (1985). These studies justify optimism that explosion generated waves for other emplacement geologies and conditions can be predicted by hydrodynamic modeling and the proposed approach can reduce uncertainties in NEM source estimates.

The computational resources needed to accurately represent hydrodynamic phenomena from explosions require extremely fine temporal and spatial discretization to model nearly instantaneous energy release and shock wave formation. Especially important is adaptive mesh refinement (AMR) to efficiently track the discontinuous shock wave fronts. These factors make it computationally challenging to calculate the response on the scales required to compare calculations with observed ground motion data recorded at distance. Fortunately two facts suggest it is unnecessary to compute motions from the elastic radius to distant receivers using expensive hydrodynamics calculations: firstly the motions in solid earth materials generally attenuate quickly to become (linear) anelastic beyond the so-called elastic radius; and secondly seismic waves observed at distance are dominated by lower frequencies (and consequently a coarse mesh is sufficient) than required for near-source shock-wave modeling. This study proposes a hybrid modeling approach where we use hydrodynamic modeling to represent the complete first principles physics of mechanical wave excitation near the explosive source volume where non-linear effects are important and pass these motions beyond the non-linear region to a linear elastic wave propagation code for much more efficient calculations of the 
seismic wave response to distant stations with a coarse mesh. We developed a methodology to pass motions from both two- and three-dimensional (2D and 3D) hydrodynamic simulations to our 3D elastic solver. In the following sections we first describe our computational methods for hydrodynamic and elastic wave motions and the one-way coupling approach. Our strategy relies on being able to compute the response from the non-linear into the linear region where motions are saved and later introduced as the source for elastic propagation. Therefore it is essential to show that hydrodynamic calculations can accurately model motions for cases involving purely elastic propagation. Then we validate the coupling strategy for a simple elastic point source and a complex non-linear source with 2D hydrodynamic calculations. We choose to use 2D axisymmetric hydrodynamic calculations instead of full 3D calculations to illustrate the coupling strategy since it is computationally less expensive to obtain direct 2D hydrodynamic solutions at distance where no analytical solutions are available (in fact one full 3D hydrodynamic to 3D elastic coupling example is also shown later to illustrate the validation and complete extensibility of the strategy). Finally, we illustrate the utility of our method by passing motions from a hydrodynamic calculation into a 3D elastic material model where scattering introduces significant complexity in the response.

\section{Computational Approach}

Complex non-linear near-source hydrodynamic phenomena have been modeled in the Lagrangian representation (e.g., Stevens et al., 1991; Stevens and Xu, 2010), Eulerian scheme (e.g.,Antoun et al., 2001) or the coupled Euler-Lagrange scheme (e.g., Brunish et al, 2011). In this study we use GEODYN, a parallel Eulerian hydrodynamics computer code developed at 
Lawrence Livermore National Laboratory (LLNL) (Antoun et al., 2001). This code incorporates many important features for modeling shock waves in geologic materials, including non-linear material constitutive properties (e.g. porosity, tensile failure, yielding, porous compaction and dilation, material strength hardening and softening as well as scale and rate-dependence, see Rubin et al., 2000; Vorobiev et al., 2007; Vorobiev, 2008), topography, gravity, and material heterogeneities. Importantly it uses adaptive mesh refinement to accurately represent the discontinuous expanding shock wave front and model the material response under rapid loading. GEODYN can be run in one-dimension (radially symmetric), two-dimensions (2D, axially symmetric) or three-dimensions (3D). GEODYN has been used on a wide range of applications involving high-energy loading of earth materials (Antoun et al., 2001, 2004; Antoun and Lomov, 2003; Lomov et al., 2003). Recently, Xu et al. (2011) used GEODYN to model elastic source spectra from buried nuclear and chemical explosions in granite and showed excellent agreement with the widely accepted empirical elastic source model of Mueller and Murphy (1971) and Stevens and Day (1985). They also showed that chemical and nuclear explosions of equivalent yield have different seismic moments with chemical explosions having higher seismic moments by a factor of about two, consistent with empirical evidence from the 1992 Nonproliferation Experiment in hard rock (Denny, 1994). GEODYN has also been used to model the near-field motions from the recent Source Physics Experiment at the Nevada National Security Site (Antoun et al., 2011).

For the linear elastic solver we utilize WPP, a parallel Carestian anelastic finite difference code also developed at LLNL (Nilsson et al., 2007; Petersson and Sjögreen, 2011). WPP has several desirable features including mesh refinement to increase the grid spacing as seismic wavespeeds 
increase with depth, free-surface topography, attenuation and absorbing boundary conditions (Appelo and Petersson, 2008; Petersson and Sjögreen, 2009, 2010; Sjögreen and Petersson, 2010). WPP has been successfully used for modeling moderate and scenario earthquake ground motions in the San Francisco Bay area (Aagaard et al., 2008, 2010; Rodgers et. al, 2008) and the effects of surface topography on explosion generated ground motions (Rodgers et al., 2010).

Seismic motions at distance have been calculated by using hybrid methods in the presence of inhomogeneities or non-linear source region response. For examples, Moczo et al. (1997) used a coupling scheme with a discrete-wavenumber method for the source and path to a finite-element method in order to incorporate the viscoelastic effects near the receiver such as a low velocity sediment filled valley. Ma et al. (2004) combined two numerical methods, the finite-element and staggered-grid finite-difference method to simulate elastic P-SV wave propagation with complicated boundary conditions. Stevens et al. (1991) and Stevens and Xu (2011) used the representation theorem to propagate motions with complex non-linear source effects to distance with elastic Green's functions. These hybrid methods involve either using analytical solutions in simplified layered structures or combining two linear numerical methods with various computational advantages.

The hybrid method proposed in this study relies on the uniqueness theorem (Aki and Richards, 1980). It uses motions (three-component velocity time-series) computed with GEODYN beyond the hydrodynamic region, i.e, in the elastic region (assuming no energy passes back to the hydrodynamic region and energy is thus one-way coupling outward) and then passes these motions to a linear anelastic solver (WPP), introduced as time-dependent boundary conditions in 
WPP. Since the free surface is traction-free, the elastic WPP solutions are uniquely determined by this velocity boundary condition on the coupling interface, warranted by the uniqueness theorem. In our approach with the uniqueness theorem only motions (velocity) are required to be saved at locations as close as possible to the nonlinear region to reduce the storage cost if needed (and results should be independent of the saving locations in the elastic region). Unlike using the representation theorem, which requires motions and tractions as well as displacement on the coupling surface (e.g. Stevens and $\mathrm{Xu}, 2011$ ), our method only requires the motions. Figure 1 shows the schematic of the strategy and the WPP domain. At each grid point (solid dots in Figure 1) of the monitoring surfaces (coupling interface) of the cube in the WPP grid setup, the Cartesian 3-components of velocity $\mathbf{v}(\mathrm{t})$ are obtained by resampling the hydrodynamic calculations in space and time, dependent upon the WPP resolution for a problem. Therefore, for 3D hydrodynamic calculations, it is a straightforward transformation from Cartesian to Cartesian systems, and for 2D hydrodynamic calculations, it is a simple procedure to transform cylindrical components (radial and vertical) to Cartesian components at the interface grid points. Again as pointed out earlier we have implemented the coupling strategy for both $2 \mathrm{D}$ and $3 \mathrm{D}$ hydrodynamic calculations coupled to WPP. Below we use 2D axisymmetric hydrodynamic calculations to validate the coupling strategy for a simple source with known analytical solutions and for a complex source without analytical solutions in which case the direct 2D solutions are conveniently obtained at distance to validate the strategy.

\section{Verification of GEODYN for Elastic Wave Propagation}


Our coupling strategy involves computing near-source motions into the elastic region with hydrodynamic methods therefore it is critical that our hydrodynamic solver be able to propagate elastic waves. Because we are interested in sources near the free surface it is essential that the hydrodynamic calculations be capable of accurately modeling surface waves. This is challenging for Eulerian methods as the motions at the surface result in mixed cells of solid earth and air. In this section we demonstrate that GEODYN is capable of propagating linear elastic waves in the presence of a free surface.

For this first verification case we chose Lamb's problem: the response of a homogeneous halfspace to a vertical point force on the surface. The compressional and shear wavespeeds of the half-space are 4000 and $2600 \mathrm{~m} / \mathrm{s}$, respectively. Analytical solutions for Lamb's problem are obtained with the method from Luco and Aspel (1983). For the vertical force, the source time function is a Ricker function with a peak frequency of $8 \mathrm{~Hz}$ and this source function has a maximum frequency of $30 \mathrm{~Hz}$. The maximum spacing is $1.25 \mathrm{~m}$ and the point-per-wavelength is about 60. Note that unlike the classical Lamb's problem with vacuum above ground, the overlying material in our case is air with density roughly 1000 times smaller than solid earth to simulate the traction-free surface (Graves, 1996). Numerical results from a 2D (axisymmetric) GEODYN calculation are plotted in Figure 2 along with the analytic solution for two locations on the free surface at 500 and $1000 \mathrm{~m}$ ranges (1.5 and 3 dominant wavelengths, respectively) and two depths (surface and 300m depth). Analytical solutions are plotted as solid lines and the numerical solutions as dashed lines in each plot. The upper and lower time-series are the vertical and radial components, respectively. As expected the surface waves dominate the response at the surface location for this type of source. The agreement of the exact solutions and numerical 
solutions at the surface locations (top 2 plots) is excellent for body waves, but there is some mismatch for Rayleigh waves in phase and amplitude. Since GEODYN relies on an Eulerian scheme, the free surface is not a clear interface between rock and air, and wave motion results in mixed cells of air and solid earth. The surface wave is very sensitive to the treatment of the free surface boundary. In this case, the imperfect implementation of the free surface might result in some energy leakage into the air above the free surface (Graves, 1996). Nonetheless the agreement between the responses computed with GEODYN and the analytic solutions are very good. The agreement between responses at the surface is better for the closer location (500 $\mathrm{m})$ as error in the surface wave response is cumulative. At the deep locations (300m deep) the body and surface waves have more nearly equal amplitudes and agreement between the GEODYN and analytic solutions is excellent as well. These results assure that GEODYN can propagate elastic waves accurately so that when motions are sampled in the elastic region they represent the true response in the presence of the free surface.

\section{Coupling the Linear Near-Source Response to Elastic Wave Propagation}

In this section we verify that the one-way coupling strategy is accurate by computing the response of an elastic medium due to an explosion source using four different approaches. In this case we consider a simple dilatational volume source (isotropic moment tensor with a step time function) at a depth of $10 \mathrm{~m}$ and compare numerical solutions computed with GEODYN and analytical solutions at offsets $360 \mathrm{~m}$ and $560 \mathrm{~m}$ on the surface. The material model is an elastic approximation to our nonlinear granite model with a compressional and shear wavespeed of 6000 and $3400 \mathrm{~m} / \mathrm{s}$, respectively. The maximum grid spacing is $0.8 \mathrm{~m}$ but along the 
wavefronts and free surface the spacing is reduced to $0.4 \mathrm{~m}$ using the adaptive mesh refinement. The results are shown on the Figure 3. All solutions were low-pass filtered below $150 \mathrm{~Hz}$ and the resolution varies from 26 to 52 points-per-wavelength. The left plots show the radial (top) and vertical (bottom) waveforms at $360 \mathrm{~m}$ and the right shows the waveforms at $560 \mathrm{~m}$ on the surface. The four solid traces in each panel represent the analytical solution, semi-analytical solution derived with reduced velocity potential (RVP), GEODYN solution and GEODYN-WPP coupling solution from top to bottom. The three dashed lines denote the differences between the three computed solutions from the analytical solution (top traces). It is evident that a buried explosion source excites stronger body waves (relative to the Rayleigh waves) than Lamb's problem (compare with Figure 2). Again we see there is an excellent agreement between the GEODYN numerical solution (third solid trace) and analytical solution (top trace) for body waves and some mismatch for Rayleigh waves at the same location, presumably due to freesurface boundary condition effects as in Lamb's problem (discussed above). In addition, for this type of volume source the reduced velocity potential (Murphy, 1991; Xu et al., 2011) is conveniently derived in the linear elastic region from the GEODYN calculation then convolved with elastic Green's functions for a point source. These semi-analytical solutions are plotted as second traces and are also in good agreement with the analytical solutions. Note that the difference between the RVP solutions and the analytical solutions is quite small as indicated by the dashed line. This serves an additional check that GEODYN yields accurate spherical waves for calculating the RVP. Finally, we use the coupling strategy described above to propagate GEODYN's solutions through the elastic region using WPP $\left(4^{\text {th }}\right.$ trace in Figure 3$)$. The grid spacing in WPP is $0.5 \mathrm{~m}$ and the resolution is about 40 points-per-wavelength for better accuracy. Note that the elastic radius in this case is just anywhere outside the source volume. It is seen that 
the body waves calculated by the coupling method match well the analytical solutions and Rayleigh waves are in phase with the analytical solutions but the amplitudes show some differences from the analytical solutions at both locations. Note that the errors (differences between the computed and analytic solutions) are largest for the GEODYN calculation to the farfield. Again it is likely attributable to the imperfect free surface implementation for elastic motions in the hydrodynamic numerical scheme. Similar to the Lamb's problem cases, the agreement at deep locations is also quite satisfactory since the wavefields are dominated by body waves (not shown). As an additional validation on the coupling scheme, we computed the nearsource wavefield due to a simple point source with 3D WPP in a linear elastic medium instead of GEODYN but saved the wavefields in a vertical plane to simulate the 2D cylindrical calculations. Then we used the same strategy as above to propagate the near-source solutions to distance. The comparison between the direct WPP solution and the WPP-to-WPP coupled solution is excellent for both body- and surface waves (not shown here) indicating that the numerical implementation of the coupling strategy is correct (3D WPP to 3D WPP coupling was not performed but results were expected to be similar to those 2D results).

\section{Coupling the Non-Linear Near-Source Response to Linear Wave Propagation}

In this section we demonstrate the coupling approach for a complex non-linear near-source calculation into linear elastic propagation. The test case is a 1 kiloton trinitrotoluene (TNT) equivalent chemical explosion at a depth of $10 \mathrm{~m}$. The material used is non-linear weathered granite (with a porosity of $1.4 \%$ ) in a half-space overlain by air. The material model was built to fit the laboratory tri-axial experiments (Chitty and Danna, 2010) with bulking and compacting 
and has lower P and S wave speeds of $3800 \mathrm{~m} / \mathrm{s}$ and $2166 \mathrm{~m} / \mathrm{s}$, respectively. No gravity is included in this hydrodynamic calculation. The chemical explosion source is represented by the JWL equation (Lee et al., 1973). As above we calculate non-linear wave propagation due to the explosion source with GEODYN, including cratering of the surface. The focus of this test case is to further illustrate that the coupling strategy functions with the complex waves generated by a nonlinear model as well as in the linear elastic cases above, and the details of the nonlinear model should not be essential to the validation. We computed the response in two ways: firstly we performed a very expensive 2D GEODYN calculation to propagate the solution to a distance of $500 \mathrm{~m}$ at the surface; secondly we use the coupling strategy to propagate the GEODYN solutions beyond the elastic radius (about $60 \mathrm{~m}$ ) to distance with WPP. For this problem no analytical solutions are available and the only way to verify the result is to compare the direct 2D GEODYN solutions with the GEODYN-to-WPP solutions. The maximum grid spacing in GEODYN is $1.4 \mathrm{~m}$ and the refinement could reduce it to $0.7 \mathrm{~m}$ on the surface and along the wavefronts, and the grid spacing in WPP is only $1.4 \mathrm{~m}$.

Figure 4 shows the resulting density distribution on the left in which the shaded region denotes the solid granite region, and the incurred nonlinear deformation on the right in which the shaded region denotes the plastic strains greater than $0.03 \%$ (darker locations correspond to higher plastic strains) at $3 \mathrm{~s}$ after the explosion. Initially the surface is at depth $=0 \mathrm{~m}$. The near-surface explosion vaporizes and ejects the surface material and produces a shallow depression to form a crater of depth $30 \mathrm{~m}$ and radius of $20 \mathrm{~m}$ along the surface (the approximate volume of the crater is about 12000 cubic meters). This near-surface explosion-induced crater is commonly seen (Gladstone and Dolan, 1977; Sublette, 2001). Note that the incurred non-linear deformation 
region is not spherically symmetric. It rather extends to about $50 \mathrm{~m}$ horizontally and vertically even further (shorter than the elastic radius $\sim 200 \mathrm{~m}$ for normally buried $1 \mathrm{kt}$ explosions in granite (Perret and Bass, 1975)). As described above, the numerical GEODYN solutions are saved at the locations beyond the non-linear deformation region starting from the dashed line at a radial distance of $64 \mathrm{~m}$ to about $100 \mathrm{~m}$, and from the depth of about $55 \mathrm{~m}$ down to $60 \mathrm{~m}$. The location of the monitoring surface in Figure 1 is usually selected close to the nonlinear region boundary to save the storage cost although the results would not vary if a larger monitoring surface were used according to the uniqueness theorem. For this particular seismic calculation the GEODYN solutions in the elastic region are resampled by interpolation in time and space, and then lowpass filtered below $30 \mathrm{~Hz}$, resulting in a minimum grid resolution of 42 points-per-wavelength for high accuracy. Higher frequencies are resolvable by using a finer mesh with the elastic code.

Figure 5 shows the comparison of the direct 2D GEODYN solutions (thick dashed line) and the WPP solutions (solid) at an offset of $500 \mathrm{~m}$ on the free surface and at a depth of $100 \mathrm{~m}$ for the $1 \mathrm{kt}$ chemical blast. The top line is the vertical component and the bottom radial component in each plot. The thin dashed lines indicate the difference of the GEODYN and GEODYN-to-WPP solutions above. The agreement of the two solutions at the surface and depth is evident in the form of the body wave and Rayleigh wave phase. The differences at late times are probably due to the imperfect outflow boundary conditions for the small volume used in these simulations. The excellent agreement in such nonlinear complex source calculations involving cratering (Figure 4) further demonstrates that the coupling scheme is adequately implemented and complex motions due to the underground explosions are appropriately propagated to distant locations where the seismic measurements are obtained to characterize the explosion sources. 


\section{Example of Scattering and Shear Wave Generation}

As an example of the utility of our coupling scheme we consider effects of a simple scatterer on 3D wave propagation at distance. The source is again $1 \mathrm{kt}$ chemical explosion at $10 \mathrm{~m}$ depth as considered in the last section and the setup is similar to Figure 4 in a half space but with a low wavespeed scattering body (200 m x $100 \mathrm{~m}$ x 50 m, with P- and S-wavespeeds of 2000 and 1155 $\mathrm{m} / \mathrm{s}$, respectively) near the receivers which is commonly seen in the realistic earth environment (e.g., Moczo et al., 1997). Figure 6a shows the experimental setup with a simple scattering body in the map view and vertical view (inset). The source location is denoted by a star, and the shaded area is the low velocity zone. The two surface stations, one at the center (station 1, the same location as in Figure 5) and the other at the edge (station 2) are marked with triangles. The same monitoring solutions obtained in Figure 5 for the homogeneous medium are passed to the linear WPP code to compute the response with the low velocity scattering body. The maximum frequency in the linear solver is $30 \mathrm{~Hz}$, corresponding to minimum wavelength about $60 \mathrm{~m}$, so it is comparable to the scattering body dimension.

The solutions without and with the low velocity scatter are compared at the two stations to illustrate the effect of the near surface low velocity zone on the wave propagation. The results are shown in Figure $6 \mathrm{~b}$ in which the solid and dashed lines represent the homogeneous and heterogeneous solutions, respectively. The left panel depicts the comparison at station 1 and the right at station 2. The radial, tangential and vertical components are plotted from bottom to top. It is seen that at the center of the scatterer (station 1) in the presence of the scattering body the 
radial and vertical components are significantly modulated: both body- and surface waves are delayed relative to the waveforms without the low velocity zone; and the waveforms become complicated and difficult to isolate seismic phases as distinct arrivals. At the edge location (station 2) however, the scattering effects are reduced in amplitudes on the radial and vertical components since the wave nearly grazes the scatter but the tangential component is pronounced and comparable to the radial and vertical components. Note that the resulting complexities seen in Figure $6 \mathrm{~b}$ show the interaction between the wavfields generated by the nonlinear source (1kt chemical explosion in this case) and the low velocity scatter, and they should be related to the source types (such as a point explosion or force source, etc). As expected, scattering from the source to the receiver plays a vital role in excitation and propagation of seismic waves and might contribute to the generation of the shear motion from explosions (e.g., Gupta and Blandford, 1983) and the current coupling scheme provides a useful pathway to investigate the full-scale wave propagation from the nonlinear explosion source to the receivers in an inhomogeneous medium.

\section{Discussion and Conclusions}

The objective of this study is to demonstrate an efficient and accurate numerical method to pass explosion-generated motions from hydrodynamic simulations to a three-dimensional linear elastic seismic wave propagation code. We demonstrated that GEODYN, our hydrodynamic code can accurately simulate elastic motions with a free surface, so that simulated motions can be confidently propagated from the non-linear region near the source to the elastic region. We 
verified GEODYN's elastic wave propagation capabilities by reproducing the analytic solutions to Lamb's problem (Figure 2) and several solutions for a shallow dilatational source (Figure 3). The accuracy of the coupling scheme from GEODYN-to-WPP is validated by comparing the solutions computed directly by GEODYN (that is computationally intensive) and the WPP coupled solutions with the GEODYN motions as tie-dependent boundary conditions in linear elastic medium (Figure 5). The agreement of the direct GEODYN solutions and GEODYN-toWPP coupled solutions at distance also checks the accuracy of GEODYN calculations. In summary, the above results demonstrate that the hydrodynamic code GEODYN is accurate and the coupling scheme from the nonlinear hydrodynamic calculations to linear elastic calculations is properly implemented.

Compared with other methods of propagating the complex near field waves generated by nonlinear explosions underground to far field, such as using the representation theorem (e.,g., Stevens and $\mathrm{Xu}, 2010$ ) in which the Green's functions are calculated in a layered structure from source to receives, the hybrid modeling method in this study provides a greater benefit that it is capable of incorporating any inhomogeneities, including topography, in the source and receiver regions, even along the propagation path. These inhomogeneous features along the propagation path impact the passing waves, making the observed seismograms at distance more complicated. As seen in the example of the low velocity scatterer (Figure 6) the waves at receivers are significantly modulated near the scatterer and the effect seems to decay with distance from the scatterer. However the tangential components are clearly quite large at the edge of the scatterer and the amplitudes are close to the radial and vertical components. Inclusion of realistic inhomogeneities will further aid in understanding the complete spectrum of wave propagation 
from the near-field to far field (e.g., Reinke and Stump, 1991) and the shear wave generation from explosions (e.g., Gupta and Blandford, 1983).

It is again worth pointing out the near-field solutions are calculated by GEODYN for the axially symmetric sources as above for the purpose of practically calculating the numerical solutions at distance and comparing with the GEODYN-to-WPP coupled solutions since the analytical solutions are unavailable for the nonlinear source calculations as in Figure 5. However, the extension to full-scale three-dimensional coupling is straightforward as described earlier although the non-linear GEODYN computation is more intensive in three-dimensions. One of the examples is shown in Figure 7 which is actually the extension to full 3D from the 2D axisymmetric case shown in Figure 3. The near field solutions in this test are calculated with GEODYN in three-dimensions and the calculations beyond the coupling interfaces are calculated with WPP in three-dimensions as usual. The two solutions from the 2D source and 3D source are superimposed in Figure 7 and the difference is denoted with the dashed lines below. The comparisons are performed on the radial (upper) and vertical (bottom) components at the two locations $360 \mathrm{~m}$ (left) and $560 \mathrm{~m}$ (right). The excellent agreement of the two solutions illustrates the accuracy of the GEODYN solutions in 3D and coupling scheme from nonlinear GEODYN to elastic WPP though no direct GEODYN solution in 3D is obtained since it is far more computationally intensive for GEODYN in 3D to calculate the seismograms at such distances. Extension to full 3D for the nonlinear explosion in Figure 4 was also performed and the agreement of the 2D and 3D is also excellent. The results are not shown here. Demonstration of this 3D GEODYN to 3D WPP coupling is important as it allows in the inclusion of near-source 
3D material heterogeneity in the GEODYN calculation, such as faults, density, yield strength, etc...

The simulation of underground explosions (chemical and nuclear) using more complete physics represented in hydrodynamic numerical codes holds great promise to improve understanding of the seismic radiation emerging from explosions. The inclusion of non-linear material properties reveals great sensitivity of seismic radiation from underground explosions to geologic material at the source (e.g. Murphy et al., 2011), as well as yield and depth-of-burial effects (e,g, Denny and Johnson, 1991) and inhomogeneities (e.g., Mandal and Toksoz, 1991; Reinke and Stump, 1991; Stevens and $\mathrm{Xu}, 2010$ ) and faults and joints as seen in the Source Physics Experiments at the NTS site (Antoun et al., 2011). This type of fully three-dimensional modeling is computationally intensive, especially in the complex source scenarios in which the time step is dictated by the source region complexities and a finer meshing is needed, however it has been made feasible with advances in high-performance computing and improvements in numerical methods to represent non-linear material response and appropriate coupling from the near-field to far-field without compromising the solutions in the seismic frequency band to understand the full spectrum of wave propagation for nuclear and chemical explosion monitoring.

\section{Data And Resources}

Only data from numerical simulations were used in this study. Hydrodynamic calculations were preformed with GEODYN. Elastic calculations were preformed with WPP, an open-source anelastic finite difference code modeling seismic waves. WPP source-code, documentation and 
examples are available at https://computation.llnl.gov/casc/serpentine/software.html (last accessed May 2012).

\section{Acknowledgements}

Simulations were performed on the SIERRA Linux cluster operated by Livermore Computing. We are grateful for access to these facilities through the Grand Challenge Allocation. We would also like to acknowledge helpful discussions with Tarabay Antoun, Ben Liu, Lew Glenn and Bill Walter. This work was performed under the auspices of the U.S. Department of Energy by Lawrence Livermore National Laboratory in part under Contract W-7405-Eng-48 and in part under Contract DE-AC52-07NA27344. This is LLNL contribution LLNL-JC-??????.

\section{References}

Aagaard, B., T. Brocher, D. Dreger, A. Frankel, R. Graves, S. Harmsen, S. Larsen, K. McCandless, S. Nilsson, N. A. Petersson, A. Rodgers, B. Sjogreen and M. L. Zoback (2008). Ground motion modeling of the 1906 San Francisco earthquake II: Ground motion estimates for the 1906 earthquake and scenario events, Bull. Seism. Soc. Amer., 98, 1012-1046, doi: 10.1785/0120060410.

Aagaard, B. T., R. W. Graves, A. Rodgers, T. M. Brocher, R. W. Simpson, D. Dreger, N. A. Petersson, S. C. Larsen and S. Ma (2010). Ground motion modeling of Hayward fault scenario earthquakes II: Simulation of long period and broadband ground motions, Bull. Seism. Soc. Amer, 100, 2945-2977.

Aki, K. and P. G. Richards (1980), Quantitative seismology: Theory and methods, by W. H. Freeman and Company. 
Antoun, T. H., I. N. Lomov and L. A. Glenn (2001). Development and application of a strength and damage model for rock under dynamic loading, proceedings of the $38^{\text {th }}$ U.S. Rock Mechanics Symposium, Rock Mechanics in the National Interest, D. Elsworth, J. Tinucci and K. Heasley (eds.), A. A. Balkema Publishers, Lisse, The Netherlands, 369374.

Antoun, T. H. and I. N. Lomov (2003). Simulation of a spherical wave experiment in marble using multidirectional damage model, $13^{\text {th }}$ American Physical Society Topical Conference on Shock Compression of Condensed Matter, Portland, OR, July 20-25, 2003.

Antoun, T. H., I. N. Lomov and L.A. Glenn (2004). Simulation of the penetration of a sequence of bombs into granitic rock, Int. J. Impact Eng., 29, 81-94.

Antoun, T., H. Xu, O. Vorobiev, and I. Lomov (2011), Initial near field predictions of the first two Source Physics Experiments (SPE), Lawrence Livermore National Laboratory Technical Report LLNL-TR-528-449, Oct.24

Appelo, D. and N.A. Petersson (2008). A stable finite difference method for the elastic wave equation on complex geometries with free surfaces, Comm. Comput. Phys., 5, 84-107.

Brunish, W., C. R. Bradley, H. J. Patton, E. E. Knight, E. Rougier, D. Steedman, A. Sussman, D. Coblentz, C. Larmat and D. Yang (2011), Source physics experiment report research in support of SPE-1, Los Alamos National Laboratory LA-UR-11-04667.

Chitty,D. E. and D. Dana, A (2010), A unified methodology for estimation of rock mass properties and associated uncertainties to support characterization of inaccessible sites, Applied Research Associates, Inc report ARA-T3-LR-1.18-05 
Denny, M. (1994). Introduction and Highlights, 1-1, Proceeding of Symposium on the NonProliferation Experiment, Rockville, Maryland

Denny, M.D and L. R. Johnson (1991). The explosion seismic source function: models and scaling laws reviewed, Explosion Source Phenomenology, pp1-24, Eds. S. R Taylor, H. J Patton and P.G Richards, Geophysical Monograph, 65.

Glasstone, S. and P. J. Dolan (1977). The Effects of Nuclear Weapons, Third Edition, United States Government Printing Office, Washington D.C.

Graves, R.W. (1996), Simulating seismic wave propagation in 3D elastic media using staggeredgrid finite difference, Bull. Seismo. Soc. Am., 86,1091-1106

Gupta, I.N. and Blandford, R.R. (1983), A mechanism for generation of short-period transverse motion from explosions, Bull. Seismo. Soc. Am., 73, 571-591

Lee, E., M. Finger and W. Collins (1973), JWL equation of state coefficients for high explosives, LLNL UCID-16189

Lomov. I. N., T. H. Antoun, J. Wagoner and J. Rambo (2003). Three-dimensional simulation of the Baneberry nuclear event, Proceedings of the $13^{\text {th }}$ American Physical Society Topical Conference on Shock Compression of Condensed Matter, Portland, OR, July $20-25$.

Luco, J.E and R.J. Apsel (1983). On the Green's functions for a layered half-space. Part I, Bull. Seismo. Soc. Am., 73, 909-929.

Ma, S, R. J. Archuleta and P. Liu (2004), Hybrid modeling of elastic P-SV wave motion: A combined finite-element and staggered-grid finite-difference approach, Bull. Seismo. Soc. Am., 94, 1557-1563 
Mandal, B. and M. N. Toksoz (1991), Effects of an explosive source in an anisotropic medium, Explosion Source Phenomenology, pp261-268, Eds. S. R Taylor, H. J Patton and P.G Richards, Geophysical Monograph, 65.

Moczo, P., E. Bystricky, J. Kristek, J. M. Carcione and M. Bouchon (1997), Hybrid modeling of P-SV seismic motion at inhomogeneous viscoelastic topographic structures, Bull. Seismo. Soc. Am, 87, 1305-1323

Mueller, R. amd J. Murphy (1971). Seismic characteristics of underground nuclear detonations Part 1: Seismic source scaling, Bull. Seismo. Soc. Am., 61, 1675-1692.

Murphy, J. (1981). P wave coupling of underground explosions in various geologic media, in Identification of Seismic source - Earthquake or Explosion, E. S. Husebye and S. Mykkelveit (eds.), p. 201-205.

Murphy, J., T. J Bennett, and B. Barker (2011), An analysis of the seismic source characteristics of explosions in low-coupling dry porous media, 524-534, Proceedings of the 2011 Monitoring Research Reviews, Tucson, Arizona

Nilsson, S., N.A. Petersson, B. Sjogreen, H.-O. Kreiss (2007). Stable difference approximations for the elastic wave equation in second order formulation, SIAMJ. Numer. Anal., 45, 1902-1936.

Perret W. R. Bass R. C. (1975). Free-field ground motion induced by underground explosions, Sandia National Laboratory Report No. SAND74-0252.

Petersson,N. A. (2011). WPP software website, https://computation.llnl.gov/casc/serpentine/software.html 
Petersson, N. A., and B. Sjögreen (2009). An energy absorbing far-field boundary condition for the elastic wave Equation, Comm. Comput. Phys. 6, 483-508.

Petersson, N. A. and B. Sjögreen (2010). Stable and efficient modeling of anelastic attenuation in seismic waves, submitted to Comm. Comput. Phys, LLNL-JRNL-460239.

Petersson, N. A., and B. Sjögreen (2011). User's guide to WPP version 2.1, Lawrence Livermore National Laboratory technical report, LLNL-SM-487431.

Reinke, R. E. and B. W. Stump (1991), Experimental studies of stochastic geological influences on near source ground motions, Explosion Source Phenomenology, pp63-72, Eds. S. R Taylor, H. J Patton and P.G Richards, Geophysical Monograph, 65.

Rodean, H (1981). Inelastic prcesses in seismic wave generation by underground explosions, in Identification of Seismic source - Earthquake or Explosion, E. S. Husebye and S. Mykkelveit (eds.), p. 97-189.

Rougier, E., H. J. Patton, E. E. Knight And C. R. Bradley (2011), Constrains on burial depth and yield of the 25 May 2009 North Korean Test from hydrodynamic simulations in a granite medium, Geophys. Res. Lett., 38, L16316, Doi:10.1029/2011g1048269.

Rodgers, A. J. E. Matzel, M. Pasyanos, A. Petersson, B. Sjogreen, C. Bono, O. Vorobiev, T. Antoun, W. Walter (2008). Seismic simulations using parallel computing and three dimensional Earth models to improve nuclear explosion phenomenology andmonitoring, 30th Monitoring Research Review, Portsmouth, VA, September 23-25, 2008.

Rodgers, A. J., O. Y. Vorobiev, N. A. Petersson, B. A. Sjogreen and M. Avants (2009a). Toward end-to-end seismic simulations for nuclear explosion monitoring, Monitoring Research Review, Tucson, AZ, September 21-23, 2009. 
Rodgers, A. J. O. Y. Vorobiev, B. Sjogreen, N. A. Petersson (2009b). Simulation of seismic waves from underground explosions in geologic media: FY2009 Progress Report, Lawrence Livermore National Laboratory Technical Report, LLNL-TR-420208.

Rodgers, A., N. A. Petersson, and B. Sjögreen (2010). Efficient generation of shear waves from shallow explosions by topographic scattering near the North Korean nuclear test site, $J$. Geophys. Res., 115, B11309, doi:10.1029/2010JB007707..

Rubin, M. B., O. Vorobiev and L. A. Glenn (2000). Mechanical and numerical modeling of a porous elastic viscoelastic material with tensile failure, Int. J. Solids and Structure, 37, 1841-1871.

Sjögreen, B. and N.A. Petersson (2010). Stable grid refinement and singular source discretization for seismic wave simulations, Comm. Comput. Phys, LLNL-JRNL-419382.

Stevens, J. L. and S. M. Day (1985). The physical basis of mb:Ms and variable frequency magnitude methods for earthquake/explosion discrimination, J. Geophy. Res., 90, 30093020.

Stevens, J. L, T. G. Baker, S. M. Day, K. L. McLaughlin, N. Rimer and B. Shkoller, Simulation of teleseismic body waves, regional seismograms, and Rayleigh wave phase shifts using two-dimensional nonlinear models of explosion sources, Explosion Source Phenomenology, pp 239-252, Eds. S. R Taylor, H. J Patton and P.G Richards, Geophysical Monograph, 65.

Stevens, J.L. and H. Xu, (2010), Wave propagation from complex 3D sources using the representation theorem, 519-528, Proceeding of Monitoring Research review, Orlando, FL. 
Sublette, C., The effects of underground explosions, http://nuclearweaponarchive.org/library/effects/undergroundeffects.html

Vorobiev O. Y., T. Antoun, I. N. Lomov, L. A. Glenn (2000). A strength and damage model for rock under dynamic loading, Proceedings of the 11th American Physical Society Topical Conference on Shock Compression of Condensed Matter, Snowbird, UT, 1999, Michael D. Furnish and Lalit C. Chhabildas and Robert S. Hixson (eds.)

Vorobiev O.Yu, Liu B.T, Lomov I.N, Tarabay T.H (2007). Simulation of penetration into porous geologic media, Int. J. of Impact Eng., 34, 721-731

Vorobiev, O. (2008). Generic strength model for dry jointed rock masses, Int. J. of Plasticity, 24, 2221-2247.

Werth, G. and R. Herst (1963). Comparison of amplitudes of seismic waves from nuclear explosions in four mediums, J. Geophys. Res., 68, 1463-1475.

Xu, H., A. J. Rodgers, I. N. Lomov, O. Y. Vorobiev (2011). Seismic source characteristics of nuclear and chemical explosions in granite from hydrodynamic simulations, submitted to Pure App. Geophys., December 2011. 


\section{Figures}

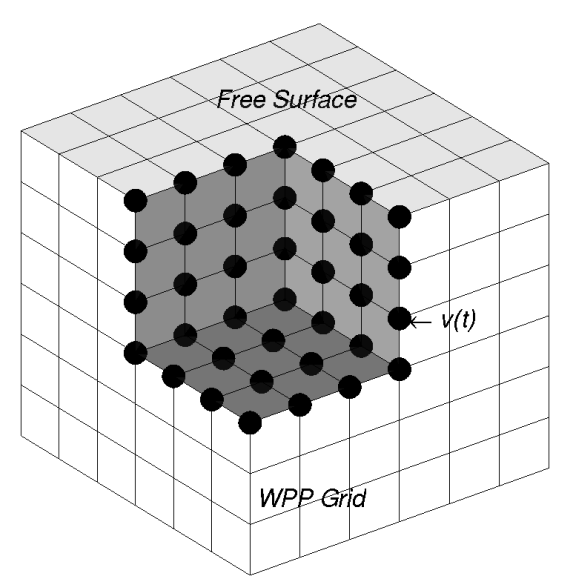

Figure 1. Schematic of the WPP grid domain and the coupling interface where at each WPP grid point, the velocity motions $\mathrm{v}(\mathrm{t})$ are passed as the driving boundary condition. 

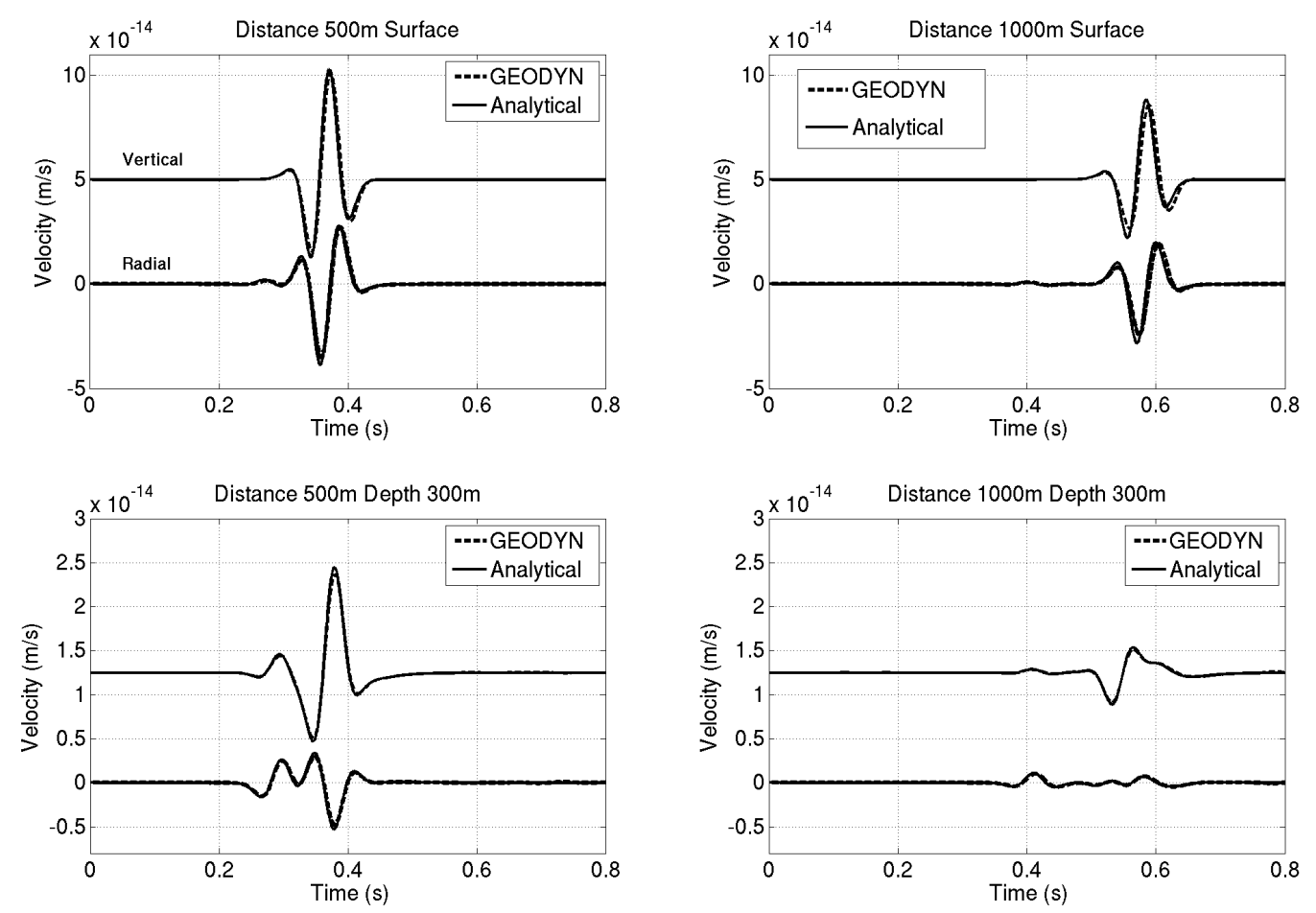

Figure 2. Computed and analytic solutions for Lamb's problem. The top and bottom traces represent vertical and radial component velocities, respectively with GEODYN (dashed) and analytical (solid) solutions at two surface locations and two deep locations. The good agreement between the numerical and analytical solutions is evident. 

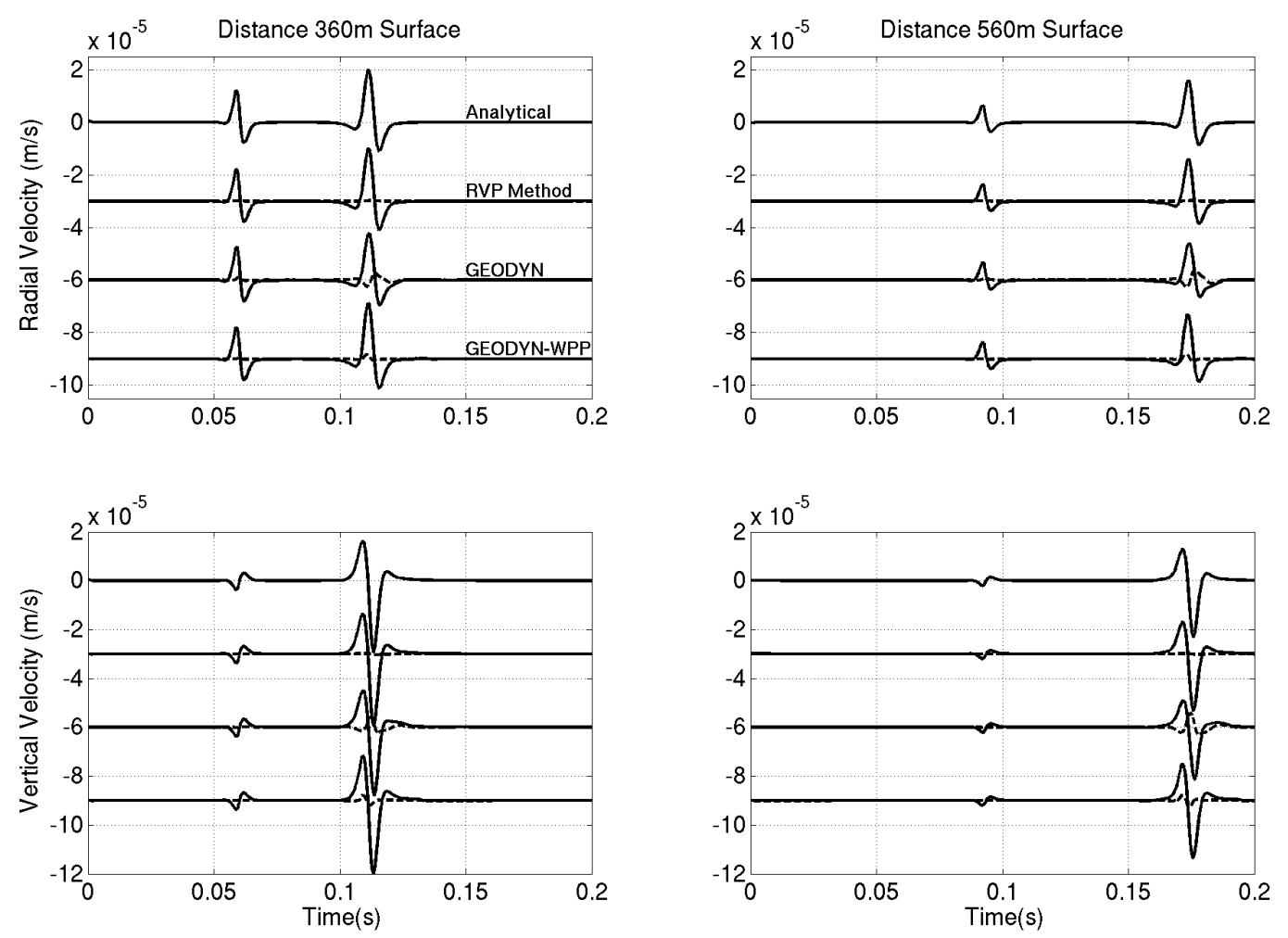

Figure 3. Computed and analytic solutions for a shallow dilatational source. The top and bottom traces represent vertical and radial component velocities, respectively. Each panel shows the analytical solution (solid, top trace), semi-analytical solution using the numerically derived reduced velocity potential (solid, $2^{\text {nd }}$ trace), direct GEODYN calculation (solid, $3^{\text {rd }}$ trace) and the WPP solutions with GEODYN solutions coupled as driving boundary conditions (solid, bottom trace). The dashed lines with each trace represent the deviation of the solution from the analytical solution (top). 

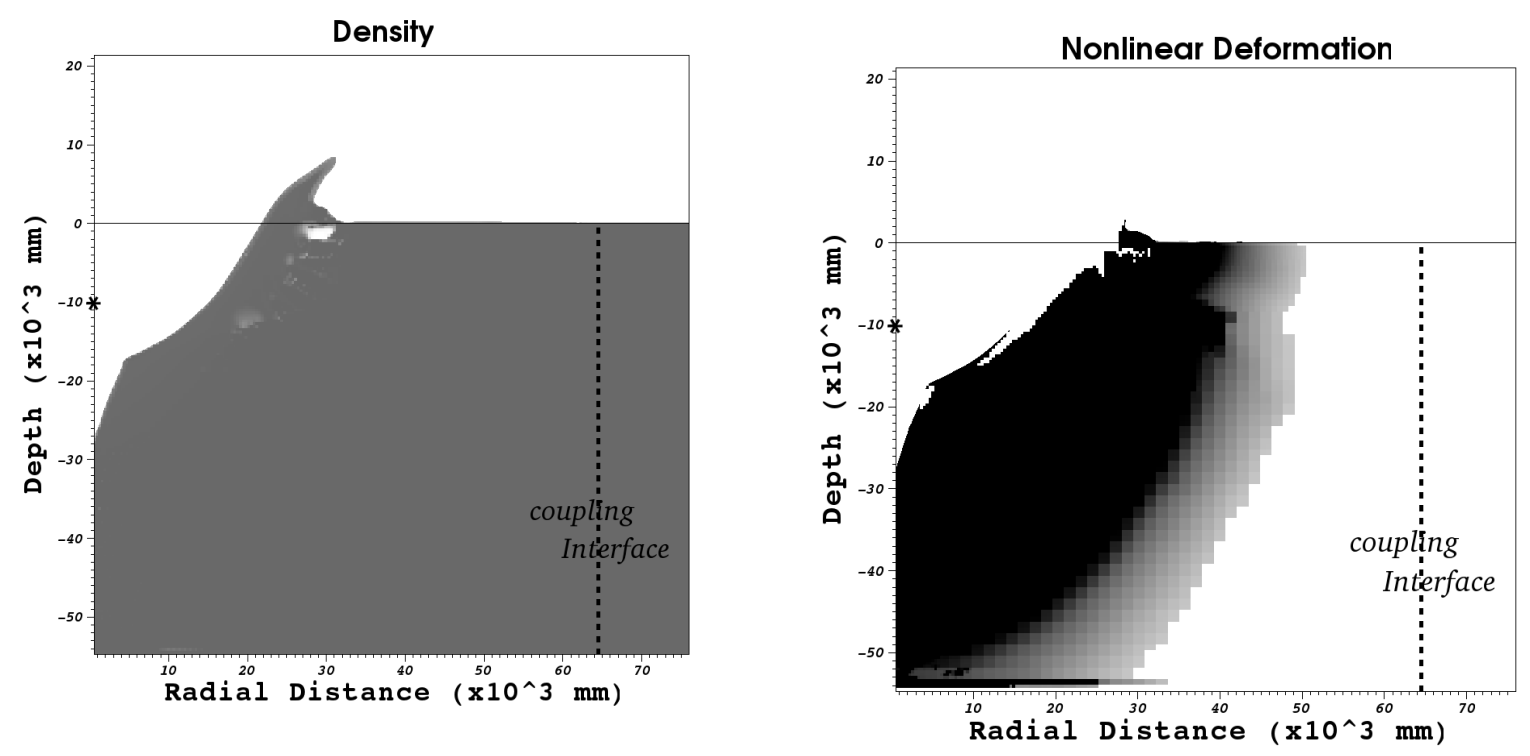

Figure 4. Explosion-induced crater formation (left, density distribution, the dark region) and nonlinear deformation (right, plastic strain $>0.03 \%$, the dark region) at $3 \mathrm{~s}$ after explosion. The free surface is initially at depth $=0$. The explosion is centered at a depth of $10 \mathrm{~m}$ (star). The crater (left) formed by a $1 \mathrm{kt}$ chemical explosion is about $30 \mathrm{~m}$ deep and $20 \mathrm{~m}$ horizontally and the nonlinear deformation extent (right) is about $50 \mathrm{~m}$. The crater volume is about 12000 cubic meters. The dashed line denotes the intersection between a vertical interface plane (Figure 1) and a radial-vertical plane (perpendicular to the vertical interface plane). 

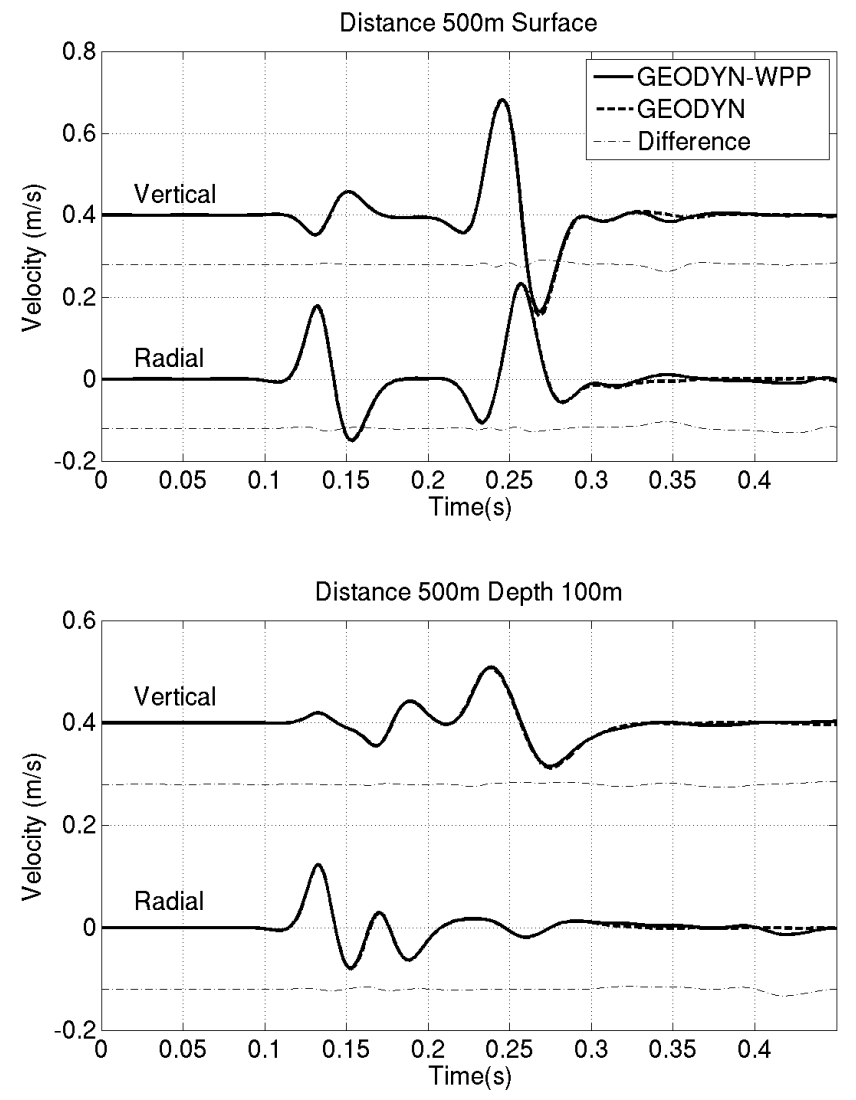

Figure 5. Comparison of the direct GEODYN solutions (thick dashed line) and WPP solutions (solid) coupled with GEODYN solutions at a surface location (top) and $100 \mathrm{~m}$ deep location (bottom). The top is the vertical component and bottom the radial component in each plot. The thin dash lines denote the difference of the two solutions above. All solutions are low-pass filtered at $30 \mathrm{~Hz}$. The agreement of the two solutions is evident in form of the body wave and Rayleigh wave phases at the two locations. 
(a)

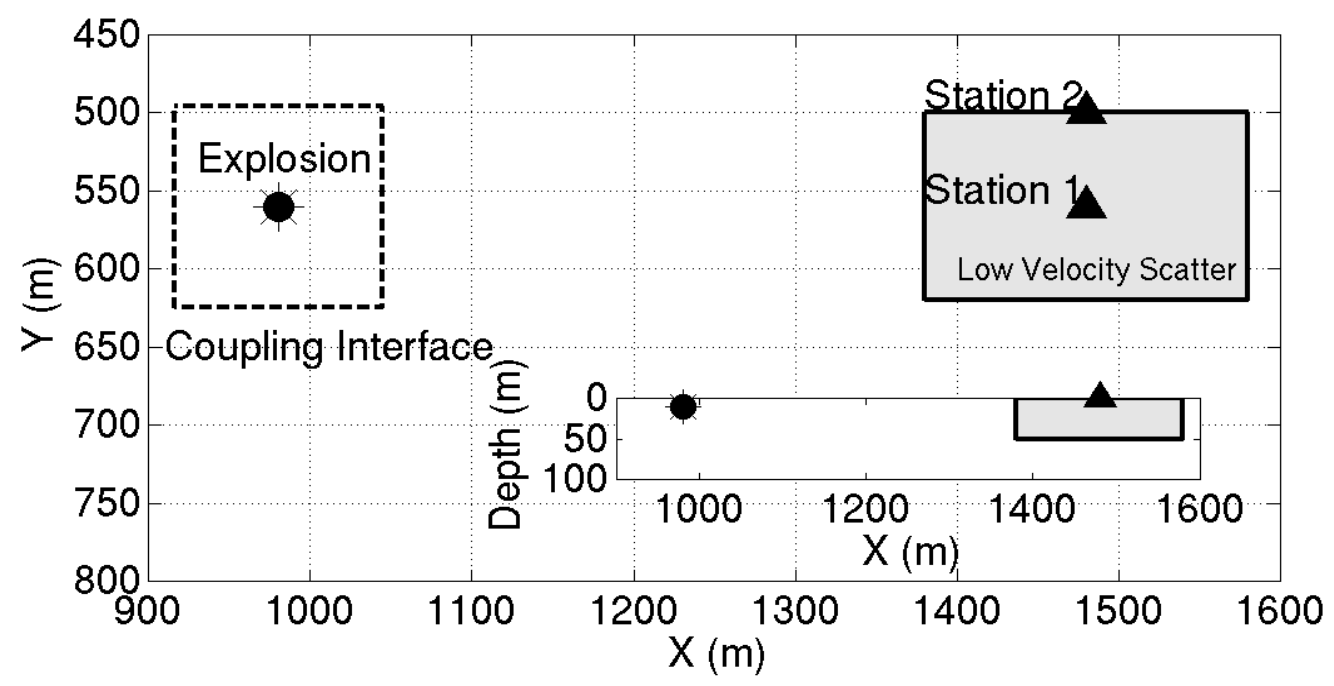


(b)
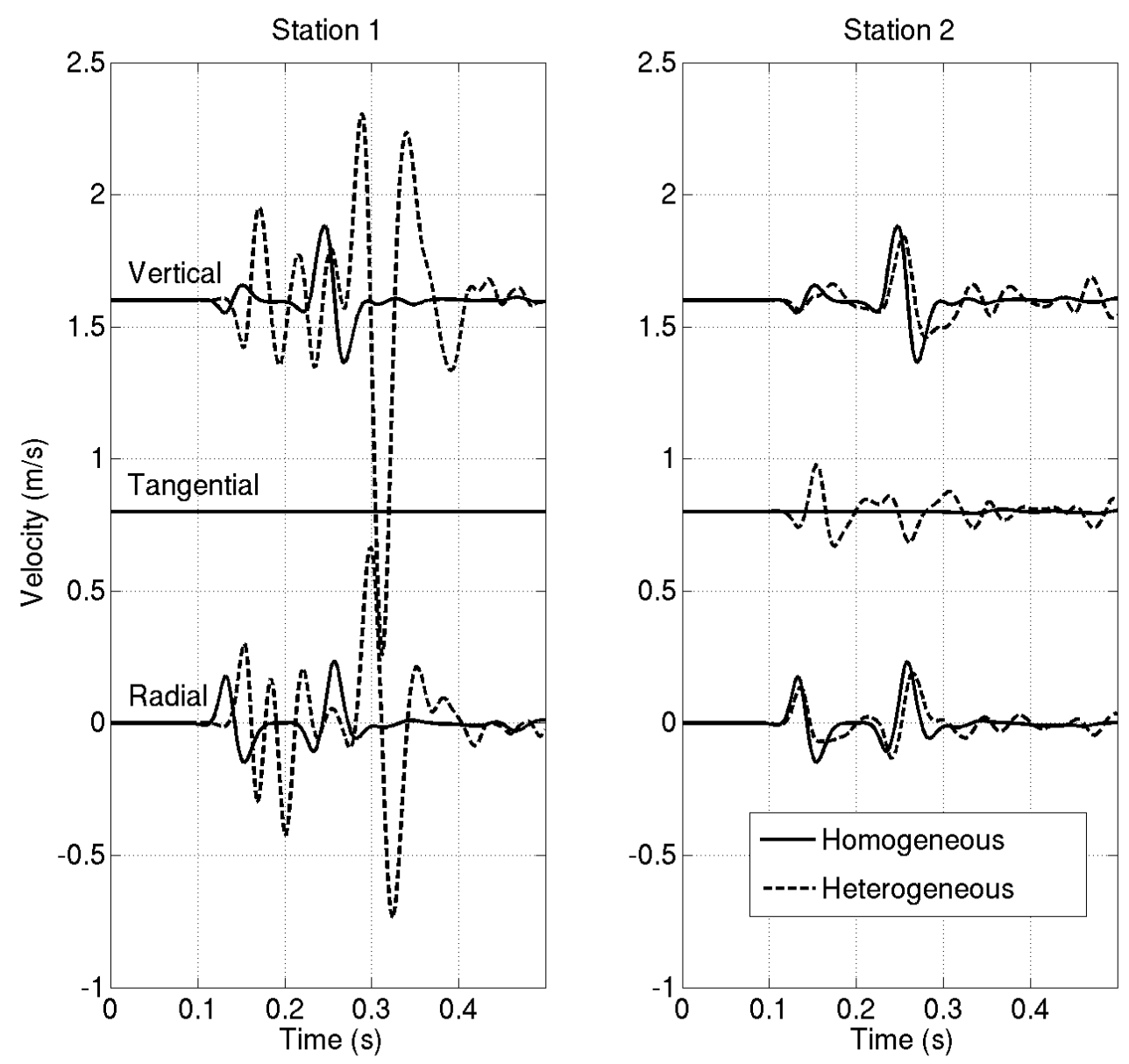

Figure 6. Comparison of the wavefields in the homogeneous and heterogeneous materials. (a) Map view and vertical view (inset) of the scatterer, explosion source and receivers. The low velocity scatterer is $400 \mathrm{~m}$ away. The stations 1 and 2 are marked with triangles. (b) Comparison of two solutions at the center of scatterer (station 1, left) and at the boundary (station 2, right), respectively, at surface. The three components, radial, tangential and vertical, are plotted from bottom to top. It is clearly seen that within the low-velocity body (Station 1) the radial and vertical components are significantly modulated and at the edge of the scatterer (Station 2) the tangential components are comparable to the radial and vertical components. 

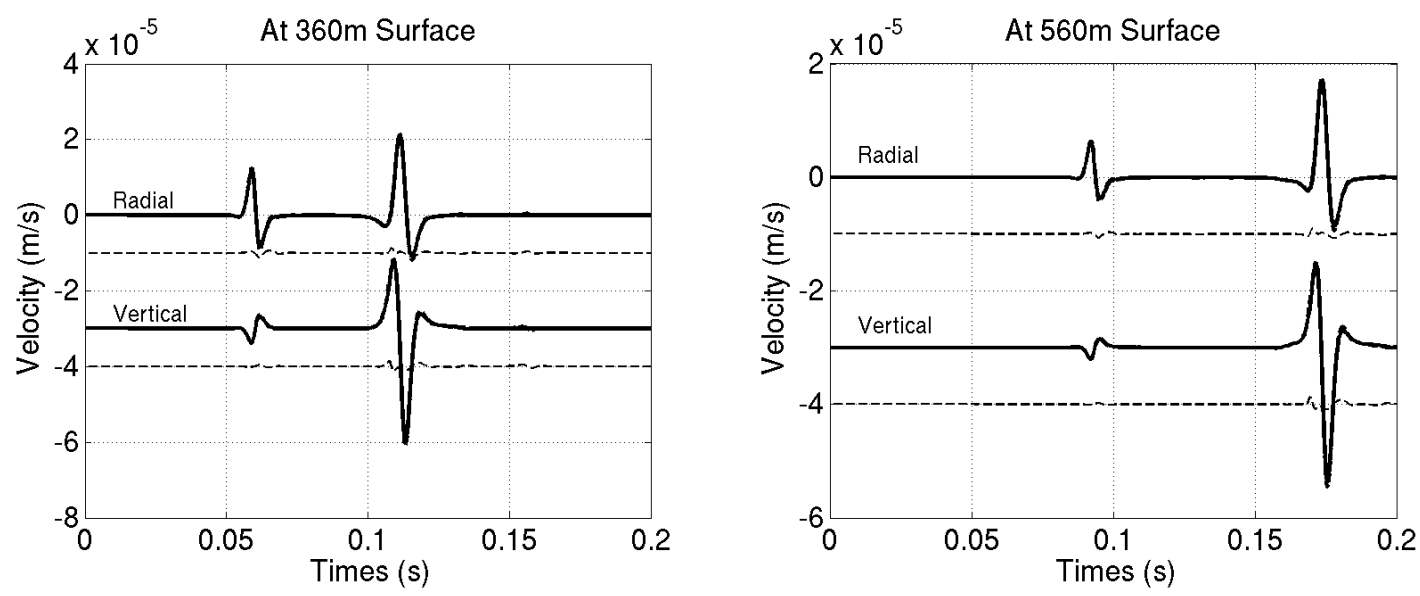

Figure 7. Comparison of the WPP solutions coupled from the 2D axisymmetric GEODYN (see Figure 3) and 3D GEODYN solutions at two offsets, $360 \mathrm{~m}$ (left) and $560 \mathrm{~m}$ (right) at surface. The two solutions are denoted in the solid lines and the difference is denoted with dashed lines below. The agreement is excellent. This demonstrates that the 3D hydrodynamic GEODYN to 3D elastic WPP coupling functions as well as the 2D to 3D coupling. 